\title{
Constituintes químicos e potencial larvicida frente Aedes aegypti do óleo essencial de Origanum vulgare $\mathrm{L}$.
}

Chemical constituents and larvicide potential against Aedes aegypti of the essential oil of the

Origanum vulgare $\mathbf{L}$.

Componentes químicos y potencial larvicida contra Aedes aegypti del aceite esencial de Origanum

vulgare $\mathbf{L}$.

Ana Patrícia Matos Pereira ORCID: https://orcid.org/0000-0003-4478-4209 Universidade Federal do Maranhão, Brasil

E-mail: ap.matos11@hotmail.com

Thércia Gabrielle Teixeira Martins ORCID: https://orcid.org/0000-0002-0506-1748

Universidade Federal do Maranhão, Brasil

E-mail: thercia.martins@hotmail.com

Ari Pereira de Araújo Neto

ORCID: https://orcid.org/0000-0001-6903-4127 Universidade Federal do Delta do Parnaíba, Brasil

E-mail: aripereiraneto@gmail.com

Carlos Eduardo Pereira Conceição ORCID: https://orcid.org/0000-0002-6735-9291

Hospital Guarás São Luís, Brasil

E-mail: dudaecarol21@gmail.com

Francilidia Oliveira Vitorino de Assunção Conceição

ORCID: https://orcid.org/0000-0002-0079-7502

Universidade Federal do Maranhão, Brasil

E-mail: francilidiaassuncao@gmail.com

Ricardo Teixeira de Sousa

ORCID: https://orcid.org/0000-0001-9304-507X Labmed e Biosaude, Brasil

Centro Universitário Internacional, Brasil

Universidade Federal do Piauí, Brasil

E-mail: ric.teix.sousa@gmail.com

Leila da Silva Silveira

ORCID: https://orcid.org/0000-0003-1050-4483

Universidade Federal do Maranhão, Brasil

E-mail: alielarcanjo@hotmail.com

Ana Maria Almeida Silva Carvalho ORCID: https://orcid.org/0000-0002-2075-5780

Universidade Federal do Maranhão, Brasil E-mail: aninha_biomed@yahoo.com.br

Liane Batista da Cruz Soares

ORCID: https://orcid.org/0000-0001-9548-3911

Universidade Federal do Maranhão, Brasil

E-mail: lianebatistadacruz@hotmail.com

Francineide Campos Aires Teixeira ORCID: https://orcid.org/0000-0002-8544-2515

Universidade Federal do Maranhão, Brasil

E-mail: francineide_ayres@ hotmail.com

Paulo Victor Serra Rosa

ORCID: https://orcid.org/0000-0003-1782-5896

Universidade Federal de Santa Catarina, Brasil

E-mail: paullovictorserra@gmail.com

Gustavo Oliveira Everton

ORCID: https://orcid.org/0000-0002-0457-914X

Universidade Federal do Maranhão, Brasil

E-mail: gustavooliveiraeverton@gmail.com 


\section{Resumo}

Os óleos essenciais extraídos de plantas medicinais têm se mostrado uma alternativa na procura de larvicidas naturais. Desta forma, o presente estudo teve por objetivo avaliar a atividade larvicida do óleo essencial extraído das folhas de Origanum vulgare L. O óleo essencial foi extraído por hidrodestilação a $100^{\circ} \mathrm{C}$ por $3 \mathrm{~h}$, com caracterização química através de Cromatografia Gasosa acoplada a espectrometria de massas (CG-EM). Em seguida, avaliou-se a letalidade do óleo essencial frente ao Aedes aegypti, calculou-se a $\mathrm{CL}_{50}$ para ação do óleo essencial a partir do método de Probit. Os constituintes majoritários encontrados no óleo essencial de $O$. vulgare foram Timol (72,14\%) e p-Cimeno $(12,25 \%)$. A atividade larvicida do óleo essencial apresentou a $\mathrm{CL}_{50}$ de $31,66 \mathrm{mg} \mathrm{L}^{-1}$. Os resultados indicam que o óleo essencial avaliado é composto por substâncias que propiciam um bom efeito larvicida, revelando sua eficiência no combate e controle do Aedes aegypti.
\end{abstract}

Palavras-chave: Bioproduto; Larvicida; Óleo essencial; Origanum vulgare.

\begin{abstract}
Essential oils extracted from medicinal plants have been shown to be an alternative in the search for natural larvicides. Thus, the present study aimed to evaluate the larvicidal activity of essential oil extracted from the leaves of Origanum vulgare L. leaves. The essential oil was extracted by hydrodistillation at $100^{\circ} \mathrm{C}$ for $3 \mathrm{~h}$, with chemical characterization through Gas Chromatography coupled to mass spectrometry (CG-MS). Then, the lethality of the essential oil against Aedes aegypti was evaluated, and the $\mathrm{LC}_{50}$ was calculated for the action of the essential oil from the Probit method. The major constituents found in the essential oil of $O$. vulgare were Timol $(72.14 \%)$ and p-cymene $(12.25 \%)$. The larvicidal activity of the essential oil presented the $\mathrm{LC}_{50}$ of $31.66 \mathrm{mg} \mathrm{L}^{-1}$. The results indicate that the evaluated essential oil is composed of substances that provide a good larvicidal effect, revealing its efficiency in combating and controlling Aedes aegypti.
\end{abstract}

Keywords: Bioproduct; Larvicida; Essential oil; Origanum vulgare.

\title{
Resumen
}

Los aceites esenciales extraídos de plantas medicinales han demostrado ser una alternativa en la búsqueda de larvicidas naturales. Así, el presente estudio tuvo como objetivo evaluar la actividad larvicidal del aceite esencial extraído de las hojas de Origanum vulgare L. hojas. El aceite esencial se extrajo por hidrodestilación a $100^{\circ} \mathrm{C}$ durante 3 h, con caracterización química mediante Cromatografía de Gases acoplada a espectrometría de masas (CG-MS). A continuación, se evaluó la letalidad del aceite esencial frente al Aedes aegypti, y se calculó la LC $\mathrm{C}_{50}$ para la acción del aceite esencial a partir del método Probit. Los principales componentes encontrados en el aceite esencial de $O$. vulgare fueron Timol $(72,14 \%)$ y p-cymene $(12,25 \%)$. La actividad larcida del aceite esencial presentó la LC 50 de $31,66 \mathrm{mg} \mathrm{L}^{-1}$. Los resultados indican que el aceite esencial evaluado está compuesto por sustancias que proporcionan un buen efecto larvicidal, revelando su eficiencia en la lucha y control del Aedes aegypti.

Palabras clave: Bioproducto; Larvicida; Aceite esencial; Origanum vulgare.

\section{Introdução}

O Aedes aegypti é o vetor conhecido por ser transmissor de vírus causadores de doenças. É um artrópode d família Diptera, Culicidae e gênero Aedes, apresenta em seu ciclo de vida as fases ovo, quatro estádios larvais, pupa e adultos, seu ciclo dura em torno de dez dias, desde o ovo até a fase adulta (Bermudi et al, 2017). A espécie possui origem africana. No Brasil, os primeiros relatos datam do final do século XIX e início do século XX. Em 1955 foi erradicado da história do Brasil, porém devidos a falhas de cobertura e ações de controle, teve seu retorno em 1976, provavelmente por meio de fronteiras e portos (Costa, 2018).

Para prevenir as doenças ocasionadas por essa espécie, tais como dengue, zika vírus e febre chikungunya, a população e a comunidade cientifica têm-se alarmado, verificando a necessidade de impedir a infecção pelo controle do vetor, reduzindo a transmissão ou bloqueando o mosquito, papel esse exercido por meio das políticas de Saúde Pública, uma vez que não existe vacina para combater o vírus transmitido (Chiarella, 2016; Braga \&Valle, 2007).

É importante salientar que a dengue está fortemente relacionada com variáveis meteorológicas. A variação sazonal da temperatura e da pluviosidade influenciam a dinâmica do vetor e a incidência da doença em todo o país (Viana \& Ignotti, 2013). O processo de dispersão do mosquito é diretamente ligado à ação do homem. Rejeitos domésticos e outros materiais descartados em lugares inadequados, crescimento urbano, clima e outros fatores propiciam a oviposição e desenvolvimento do vetor e, com isso, aumento da transmissão de doenças (Ribeiro, 2013). 
O controle vetorial é realizado através da eliminação de locais para oviposição ou a eliminação das larvas (Coller, 2011), utilizando inseticidas, temefós, melathion e fenitrothion, constitui a principal medida adotada pelos Programas de Saúde Públicas (Silva et al., 2017). O uso constante desses produtos sintéticos tem ocasionado diversos danos ao meio ambiente, seja no ar ou na água, interferindo diretamente na saúde do ser humano, além de ocasionar vários transtornos ambientais, sociais e econômicos (Yanola et al., 2015).

Quando abordamos sobre as condições de saúde da população, as preocupações viram interesse de todos, uma vez que o ambiente onde se vive vai interferir significativamente na sua qualidade de vida (Júnior Santos et al., 2020). Posto isto, surge então a necessidade de métodos alternativos, principalmente aqueles baseados em recursos naturais, sendo o inseticida sustentável, ecologicamente correto, eficaz, possuir baixa toxicidade aos mamíferos e não devendo modificar de forma significativa as características da água (Dias \& Moraes, 2014).

Estudos evidenciaram que diversas plantas produtoras de óleo essencial podem ser utilizadas através do seu efeito larvicida sobre o Aedes aegypti. Os produtos à base de plantas, além de terem efeito inseticida comprovado, apresentam uma diversidade de compostos ativos, os quais agem sinergicamente, apresentando características atraentes, desalojantes ou repelentes, entre outras que podem ser empregados em sistemas de manejo integrado de pragas, como alternativas dirigidas para controle e monitoramento das populações de insetos (Sousa et al., 2019).

Os óleos essenciais podem ser obtidos a partir da planta inteira ou parte dela, por meio de extração com diferentes tipos de solventes, podendo ter toxicidade para as formas adultas do mosquito (inseticida), para os estágios larvais (larvicidas), inibindo o crescimento e diferenciação das mesmas, bem como inibindo a maturação e/ou eclosão dos ovos (ovocida) (Takagi, 2020). Os óleos essenciais apresentam uma composição relativamente complexa, podendo possuir entre dezenas a centenas de compostos, e em geral o fitoconstituinte em maior concentração é aquele que atribui à atividade biológica do óleo essencial (Cunha et al., 2012). Estes compostos bioativos fazem parte da constituição de diferentes grupos químicos como: hidrocarbonetos, álcoois, ésteres, aldeídos, cetonas, fenóis e terpenos sendo que, os compostos terpênicos são considerados os mais abundantes (Felipe\&Bicas, 2017).

Dentre as plantas produtoras de óleo essencial, destaca-se Origanum vulgare. É uma especiaria originada do Mediterrâneo, pertencente a família Lamiaceae, com sabor e aromas característicos (Porto \& Rosa, 2018). O uso de longa data do $O$. vulgare na medicina tradicional atrai ainda mais interesse no projeto de novas formulações farmacêuticas em várias áreas, investigações têm mostrado alto potencial biológico (Oniga et al., 2018).

Desta forma, o presente estudo tem por objetivo determinar os constituintes químicos e atividade larvicida frente ao Aedes aegypti do óleo essencial de Origanum vulgare, visando obter através de um produto natural, uma alternativa segura e eficiente no controle e combate da população do Aedes aegypti.

\section{Metodologia}

\subsection{Plant material}

A coleta do material vegetal utilizado nesta pesquisa foi realizada de outubro a dezembro de 2019. Folhas de $O$. vulgare foram coletadas no município de São Luís, Brasil. As amostras foram depositadas no Herbário Ático Seabra da Universidade Federal do Maranhão. Após a coleta, as espécies vegetais foram transportadas para o Laboratório de Pesquisa e Aplicação de Óleos Essenciais (LOEPAV/UFMA).

\subsection{Obtenção do óleo essencial}

Para a extração do OE, a técnica de foi utilizada com um extrator de vidro Clevenger acoplado a um balão de fundo 
redondo acoplado a uma manta elétrica como fonte geradora de calor. Foram utilizados $100 \mathrm{~g}$ do material vegetal, adicionando água destilada (1:10). A hidrodestilação foi realizada a $100^{\circ} \mathrm{C}$ durante $3 \mathrm{~h}$ e o OE extraído foi coletado. Cada OE foi seco por percolação com sulfato de sódio anidro $\left(\mathrm{Na}_{2} \mathrm{SO}_{4}\right)$. Essas operações foram realizadas em triplicados e as amostras armazenadas em ampolas de vidro âmbar sob refrigeração de $4^{\circ} \mathrm{C}$. Posteriormente submetidas às análises.

\subsection{Análises de constituintes químicos}

Os constituintes dos OEs foram identificados por cromatografia gasosa acoplado à espectrometria de massa (GC-MS). $1,0 \mathrm{mg}$ da amostra foi dissolvida em $1000 \mu \mathrm{L}$ de diclorometano (pureza 99,9\%). O programa AMDIS (Automated Mass Spectral Deconvolution Mass \& Identification System) foi utilizado para identificar os compostos da amostra.

\subsection{Atividade larvicida}

Os ovos foram coletados na Universidade Federal do Maranhão, Campus Bacanga, em São Luís/MA, por meio de armadilhas chamadas ovitrampas. Estas consistem em recipientes marrons $(500 \mathrm{~mL})$, polietileno, com $1 \mathrm{~mL}$ de levedura cervejeira e $300 \mathrm{~mL}$ de água corrente e duas Palhetas de Eucatex para a ovoposição do mosquito. As armadilhas foram inspecionadas semanalmente para substituição das palhetas e coleta de ovos e enviadas ao Laboratório de Pesquisa e Aplicação de Óleos Essenciais da Universidade Federal do Maranhão - UFMA.

Os ensaios para a atividade larvicida foram realizados de acordo com a metodologia adaptada proposta por Silva (2006). Inicialmente, foi preparada uma solução mãe de $100 \mathrm{mg} \mathrm{L}^{-1}$ de OE diluída em $2 \%$ de dimetilsulfóxido (DMSO). A partir desta solução, cinco diluições foram preparadas nas concentrações 10, 20, 50, 70 e $100 \mathrm{mg}$ L-1. Foram adicionadas dez larvas em cada concentração na proporção de $1 \mathrm{~mL} / \mathrm{larva}$.

Todos os testes foram realizados em triplicatas e como controle negativo foi utilizada uma solução formada de DMSO $10 \%$, e como controle positivo, uma solução de temefós a 100 ppm, equivalente à concentração utilizada pela Fundação Nacional de Saúde (Funasa) para o controle larvicida do vetor. Após 24 horas, os vivos e os mortos foram contados, e as larvas que não reagiram ao toque foram consideradas mortas após 24 horas do início do experimento. As taxas de mortalidade foram obtidas pela média de indivíduos mortos em função do logaritmo da dose testada. A análise estatística dos dados para o $\mathrm{CL}_{50}$ foi realizada de acordo com Probit (1952).

\section{Resultados e Discussão}

\subsection{Constituintes químicos}

De acordo com os resultados obtidos através da Cromatografia Gasosa acoplada a Espectrometria de Massas (CG/EM) o composto majoritário do óleo essencial das folhas de Origanum vulgare L. foi o timol $(72,14 \%)$, seguido do pcimeno $(12,25 \%)$.

O teor de timol $(72,14 \%)$ relatado neste estudo torna-se significante ao compararmos com Vazirian et al. (2015) que ao extraí-lo das folhas de $O$. vulgare coletadas em Noshahr, Mazandaran Província (Irã) realizou a caracterização química do mesmo através de CG/EM e notou a presença do constituinte em 37,13\% de sua amostra, enquanto um teor de 3,58\% de pcimeno.

Oliveira et al. (2021) também detectaram teor de timol inferior ao deste estudo, apresentando 10,4\% do OE extraído das partes aéreas de Origanum vulgare L. No entanto, resultados semelhantes foram encontrados por Fikry et al. (2019), ao realizarem a caracterização quimica de óleos essenciais de $O$. vulgare do México e do Brasil, detectaram a presença de timol, g-terpineno e carvacrol, como seus constituintes majoritários. Apesar da metodologia de extração semelhantes, há variações na composição química de óleos essenciais. 
O timol é um monoterpeno, pertencente à família Lamiaceae e encontrado em grandes proporções principalmente nos óleos essenciais de tomilho e orégano (Lemos et al., 2017). Esse composto se destaca em estudos com produtos naturais de espécies vegetais por suas propriedades biológicas antibacterianas, antifúngicas, antiparasitárias, inseticidas e repelentes (Belato et al., 2018; Marchese et al. 2016; Nascimento, 2017). Assim, é de vital importância o estudo do OE extraído de $O$. vulgare como uma fonte natural significativa de timol tanto para aplicações biológicas quanto para as indústrias em geral.

\subsection{Atividade Larvicida}

A Tabela 1 apresenta a mortalidade das larvas de Aedes aegypti nas concentrações do óleo essencial testadas com cálculo da $\mathrm{CL}_{50}$ e parâmetros estatísticos pelo método de Probit.

Tabela 1 - Mortalidade Aedes aegypti para ação do óleo essencial pelo método Probit.

\begin{tabular}{|c|c|c|c|c|c|c|}
\hline $\log C$ & Mortality & $\mathrm{CL}_{50} \mathrm{mg} \mathrm{L}^{-1}$ & $\mathrm{CL}_{90} \mathrm{mg} \mathrm{L}^{-1}$ & $x^{2}$ & $\sigma$ & $\overline{\mathbf{R}^{2}}$ \\
\hline 1,00 & 13,1 & \multirow{9}{*}{$\begin{array}{c}31,66 \\
(23,64-42,40)\end{array}$} & \multirow{9}{*}{$\begin{array}{c}87,58 \\
(\mathbf{7 6 , 8 4 -} 95,60)\end{array}$} & \multirow{9}{*}{1,000} & \multirow{9}{*}{0,427} & \multirow{9}{*}{0,950} \\
\hline 1,30 & 35,9 & & & & & \\
\hline 1,48 & 52,3 & & & & & \\
\hline 1,70 & 55,5 & & & & & \\
\hline 1,78 & 68,6 & & & & & \\
\hline 1,85 & 78,4 & & & & & \\
\hline 1,95 & 81,7 & & & & & \\
\hline 2,00 & 91,5 & & & & & \\
\hline 2,08 & 94,7 & & & & & \\
\hline
\end{tabular}

Fonte: Autores.

Através da Tabela 2, observa-se que o OE apresentou $\mathrm{CL}_{50}$ de 31,66 $\mathrm{mg} \mathrm{L}^{-1}$ e $\mathrm{CL}_{90}$ de 87,58 $\mathrm{mg} \mathrm{L}^{-1}$ contra as larvas do mosquito Aedes aegypti, sendo classificado como altamente ativo de acordo com Cheng et al. (2003), que considera bons agentes larvicidas as substâncias com valor de $\mathrm{CL}_{50}$ inferior a $100 \mathrm{mg} \mathrm{L}^{-1}$.

Esse resultado é confirmado por de Oliveira et al. (2021), ao avaliar o potencial larvicida de O. vulgare (L.), obtiveram $\mathrm{CL}_{50}$ de $37,5 \mathrm{mg} \mathrm{L}^{-1}$, frente as larvas do mosquito Aedes aegypti. Vale ressaltar escassez de estudos na literatura sobre a atividade larvicida do óleo essencial de $O$. vulare L. frente larvas de Ae. aegypti.

Em pesquisa realizada por Chaves et al. (2020), sobre a avaliação da atividade larvicida do OE de outra espécie de Origanum (O. majorana L.), alcançaram $\mathrm{CL}_{50}$ de $62,81 \mu \mathrm{g} \mathrm{mL}^{-1}$, após $24 \mathrm{~h}$ de exposição das larvas ao $\mathrm{OE}$, apresentando potencial larvicida.

De acordo com Cantrell et al. (2010), as substâncias larvicidas atuam através do trato respiratório ou gastrointestinal, por absorção. Visto que, quando absorvidas pelas larvas, podem causar efeitos sistêmicos em diversos órgãos ou atingir o local de ação.

O potencial larvicida do OE em estudo, pode ser atribuído a seus compostos químicos majoritários, os monoterpenos timol e p-cimeno. Dessa forma, alguns estudos mostraram um efeito significativo de p-cimeno e timol, frente larvas de duas espécies de Aedes (Aedes aegypti e Aedes albopictus), com $\mathrm{CL}_{50}$ de 


\section{Conclusão}

Em conformidade com os resultados obtidos, foi possível classificar o óleo essencial de Origanum vulgare como moderadamente tóxico, em razão de apresentar a CL50 de 199,5 mg L-1. Determinou-se o Timol (72,14\%) e P-cimeno (12,25\%) como sendo os constituintes majoritários e atribuiu-se a eles a ação larvicida do óleo essencial em estudo, onde apresentou ação larvicida altamente eficiente, por manter a $\mathrm{CL}_{50}$ abaixo de $50 \mathrm{mg} \mathrm{L}^{-1}$. À vista disso, conclui-se que o óleo essencial é composto por substâncias que incentivam sua aplicação, em virtude do seu potencial biológico larvicida.

\section{Referências}

Belato, K. K., de Oliveira, J. R, de Oliveira, F. S., de Oliveira, L. D., \& Camargo, S. E. A. (2018). Citotoxicidade e genotoxicidade do timol verificadas em macrófagos murinos (RAW 264.7) após análise antimicrobiana em Candida albicans, Staphylococcus aureus e Streptococcus mutans. Journal of Functional Foods , $40,455-460$.

Bermudi, P. M. M., Kowalski, F., Menzato, M. M., Ferreira, M. D. C., Passos, W. B. S. D., Oku, V. J. A., ... \& Chiaravalloti Neto, F. (2017). Aedes aegypti breeding site in an underground rainwater reservoir: a warning. Revista de saude publica, 51, 122.

Cantrell, C. L., Pridgeon, J. W., Fronczek, F. R., \& Becnel, J. J. (2010). Structure-activity relationship studies on derivatives of eudesmanolides from Inula helenium as toxicants against Aedes aegypti larvae and adults. Chemistry \& biodiversity, 7(7), 1681-1697.

Cheng, S. S., Chang, H. T., Chang, S. T., Tsai, K. H., \& Chen, W. J. (2003). Bioactivity of selected plant essential oils against the yellow fever mosquito Aedes aegypti larvae. Bioresource Technology, 89(1), 99-102.

Coller, B. A. G., \& Clements, D. E. (2011). Dengue vaccines: progress and challenges. Current opinion in immunology, 23(3), $391-398$.

Costa, J. (2018). Potencial uso da manipueira no controle larvicida do Aedes aegypti.

Cunha, G. H. (2012). Efeito farmacológico das fraçõeshexânica, clorofórmica e metanólica do óleo essencial da Alpinia zerumbet na reatividade vascular in vitro e nos parâmetros cardiovasculares in vivo. (Tese de doutorado, Universidade Federal do Ceará).

Dias, C. N., \& Moraes, D. F. C. (2014). Essential oils and their compounds as Aedes aegypti L. (Diptera: Culicidae) larvicides. Parasitology research, 113(2), $565-592$.

Felipe, L. O. \& Bicas, J. L. (2017). Terpenos, aromas e a química dos compostos naturais. Química Nova na Escola, 39(2), 120-130.

Fikry, S., Khalil, N., \& Salama, O. (2019). Perfil químico, dinâmica bioestática e biocida do óleo essencial de Origanum vulgare L. AMB Express , 9 (1), 1-10.

Huang, H. T., Lin, C. C., Kuo, T. C., Chen, S. J., \& Huang, R.N. (2019). Composição fitoquímica e atividade larvicida de óleos essenciais de plantas fitoterápicas. Planta, 250, 59-68.

Júnior, P. S. S., Everton, G. O., Rosa, P. V. S., dos Santos Souza, L., de Assunção Conceição, F. O. V., da Cruz Soares, L. B., ... \& Mouchrek Filho, V. E. (2020). Atividade larvicida do óleo essencial de Alpinia zerumbetfrente as larvas do mosquito Aedes aegypti. Research, Society and Development, 9(8), e194985578-e194985578.

Lemos, M. F., Lemos, M. F., Pacheco, H. P., Guimarães, A. C., Fronza, M., Endringer, D. C., \& Scherer, R. (2017). Seasonal variation affects the composition and antibacterial and antioxidant activities of Thymus vulgaris. Industrial Crops and Products, 95, 543-548.

Marchese, A., Orhan, I. E., Daglia, M., Barbieri, R., Di Lorenzo, A., Nabavi, S. F., ... \& Nabavi, S. M. (2016). Antibacterial and antifungal activities of thymol: A brief review of the literature. Food chemistry, 210, 402-414.

Oliveira, A. A, França, L. P, Ramos, A. D. S., Ferreira, J. L. P., Maria, A. C. B., Oliveira, K. M., ... \& de Andrade, S. J. R. (2021). Atividades larvicida, adulticida e repelente contra Aedes aegypti L. de duas especiarias comumente usadas, Origanum vulgare L. e Thymus vulgaris L. South African Journal of Botany, $140,17-24$.

Oniga, I., Pușcaş, C., Silaghi-Dumitrescu, R., Olah, N. K., Sevastre, B., Marica, R., ... \& Hanganu, D. (2018). Origanum vulgare ssp. vulgare: Composição química e estudos biológicos. Molecules, 23 (8), 2077.

Nascimento, G. J. (2017). Estudo da atividade inseticida e repelente do timol. Trabalho de conclusão de curso, Biotecnologia do Centro de Biotecnologia, Universidade Federal da Paraíba - UFPB.

Porto, L. L., \& Rosa, L. R. V. D. (2018). Avaliação do potencial antimicrobiano de óleos essenciais de coentro (coriandrum sativum l.) e orégano (origanum vulgare l.) (Bachelor's thesis, Universidade Tecnológica Federal do Paraná).

Ribeiro, M. S. (2013) Análise comparativa entre as metodologias de monitoramento da infestação do Aedes aegypti, associadas à transmissão de dengue nos municípios de Itaboraí e Guapimirim, Rio de Janeiro. 93f. Dissertação (Mestrado em Saúde Pública) - Fundação Oswaldo Cruz, Escola Nacional de Saúde Pública Sergio Arouca, Rio de Janeiro.

Sheng, Z., Jian, R., Xie, F., Chen, B., Zhang, K., Li, D., ... e Hong, W. D. (2020). Rastreamento da atividade larvicida de 53 óleos essenciais e seu efeito sinérgico para a melhoria da eficácia da deltametrina contra Aedes albopictus. Culturas e produtos industriais, 
Research, Society and Development, v. 10, n. 9, e9910917683, 2021

(CC BY 4.0) | ISSN 2525-3409 | DOI: http://dx.doi.org/10.33448/rsd-v10i9.17683

Silva, T. I., Alves, A. C. L., de Azevedo, F. R., Marco, C. A., dos Santos, H. R., \& Alves, W. S. (2017). Efeito larvicida de essenciais de plantas medicinais sobre larvas de Aedes aegypti L. (Diptera: Culicidae). Revista Verde de Agroecologia e Desenvolvimento Sustentável, 12 (2), 256-260.

Sousa, D. A. D. (2019). Avaliação da atividade larvicida de óleos essenciais de espécies de citrus frente às larvas de Aedes aegypti.

Szczepanik, M., Zawitowska, B., \& Szumny, A. (2012). Insecticidal activities of Thymus vulgaris essential oil and its components (thymol and carvacrol) against larvae of lesser mealworm, Alphitobius diaperinus Panzer (Coleoptera: Tenebrionidae). Allelopathy Journal, 30(1), 129-142.

Takagi, B. A., de Souza, T. G. B., de Oliveira, M. D., Bernardes, L. G., Oda, J. Y., Machado, A. R. D. S. R., \& Machado, A. M. (2020). Efeito larvicida e ovocida de extratos de Crotalaria pallida sobre o vetor Aedes aegypti. Brazilian Journal of Development, 6(5), 23060-23074.

Vazirian, M., Mohammadi, M., Farzaei, M., Amin, G., Amanzadeh, Y. (2015). Composição química e atividade antioxidante de Origanum vulgare subsp. óleo essencial vulgare do Irã. Research Journal of Pharmacognosy, 2 (1), $41-46$.

Viana, D. V., \& Ignotti, E. (2013). The ocurrence of dengue and weather changes in Brazil: a systematic review. Revista Brasileira de Epidemiologia, 16(2), 240-256.

Yanola, J., Chamnanya, S., Lumjuan, N., \& Somboon, P. (2015). Insecticides resistance in the Culex quinquefasciatus populations from northern Thailand and possible resistance mechanisms. Acta tropica, 149, 232-238. 